besten Reactionen gehört, zu verlassen, wie diess $\mathrm{Huizinga}{ }^{*}$ ) will mo dafür Molybdänsäure anräth, sondern Ma $\mathrm{I}$ yürde vur folgende Cautelen damit in Verbindung bringen. Der Farbstofi ist aus der jeweiligen Flüssigkeit (Harn etc.) durch Digeriren mit Thierkohle wegzunehmen; der Kupfervitriol ist der alkalisch gemachten Flüssigkeit so lange hinzuzusetzen, dass ein kleiner Theil Kupferhydroxyd noch ungelöst ist, und dann wäre bei nochmaligem Erhitzen auf die Gelbfärbung des hinzugebrachten Zinkoxyds zu achten. Die Empfindlichkeit einer reinen Zuckerlösung auf Kupfersalze ist aber dabei nicht zu erreichen.

*) Pflüger's Archiv 1871.

\title{
Berichtigungen.
}

Im ersten Hefte dieses Jahrgangs pag. 124 Zeile 4 von oben lies $\mathrm{S} c h o ̈ n r$ anstatt $\mathrm{Schöme.} \mathrm{Der} \mathrm{gleiche} \mathrm{Druckfehler} \mathrm{ist} \mathrm{auch} \mathrm{in} \mathrm{dem} \mathrm{Inhaltsverzeichnisse}$ des genannten Heftes zu corrigiren.

Im zweiten Hefte dieses Jahrganges S. 257 ist die Zahl 35,918 als das von Dr. Gl. Winkler ermittelte Atomgewicht des Indiums angegeben. Derselbe hat diese Zahl allerdings 1864 bei seinen ersten, mit 'wenigem Material ausgeführten Untersuchungen üher das Indium gefunden, dieselbe aber bei späteren Arbeiten selbst als unrichtig erkannt und 1867 fünf neue Bestimmungen publicirt, welche im Mittel anf die Zahl 37,813 führten (Journ. f. prakt. Chem. 102. 282). Diese Zahl stimmt sehr nahe mit dem ron $B$ unsen gefundenen Atomgewichte überein, und würde man, wenn man jene Zahl - anlässlich der interessanten Beobachtungen Bunsen's über die specifische Wärme des.Indiums - anderthalbmal so gross annimmt, In $=56,719$ erhalten.

D. Red.

\section{Chemisches Laboratorium und Pharmaceutische Lehr-Anstalt zu Wiesbaden.}

Das chemische Iraboratorium verfolgt wie bisher den Zweck, junge Märner, welche die Chemie als Haupt- oder Hilfsfach erlernen wollen, aut's Gründlichste in diese Wissenschaft einzufuihren und mit ihrer Anwendung im praktischen Leben bekannt zu machen, - die pharmaceutische Lehranstalt ist bestimmt, jungen Pharmaceuten, welche in ibrem Fache bereits praktisch erfahren sind, eine gründliche und umfassende wissenschaftliche Ansbildung in den Naturwissenschaften und der Pharmacie zu geben und denselben namentlich auch Gelegenheit zu bieten, sich mit allen Theilen der praktischen Chemie tiichtig vertrant zu machen.

Der Besuch der pharmaceutischen Lehranstalt (während $1^{1 / 2}, 2$ oder 3 Semestern) wird nach Verfügung des Herrn Ministers der geistlichen, Unterrichts- und Medieinal-Angelegenheiten, d. d. Berlin 20.Juli 1867, beim Preussischen Staats-Examen den Pharmaceuten gleich einem Servirjahr angerechnet.

Der Sommer-Cursus beider Anstalten beginnt am 24. A pril, der WinterCursus am 15. Oetober.

Statuten und Vorlesungs-Verzeichniss sind durch C. W. Kreidel's Verlag in Wiesbaden oder durch den Unterzeichneten unentgelttich zu beziehen.

Wiesbaden.

Dr. R. Fresenins, Geh. Hofrath und Professor. 